\title{
MSDM: Maximally Spatial Disjoint Multipath Routing Protocol for MANET
}

\author{
Wesam Almobaideen, Roba Al-Soub, Azzam Sleit \\ Computer Science Department, King Abdulla II School for Information Technology, \\ The University of Jordan, Amman, Jordan \\ Email: wesmoba@ju.edu.jo,roba_soub@yahoo.com,azzam.sleit@ju.edu.jo
}

Received August 23, 2013; revised September 20, 2013; accepted September 27, 2013

Copyright (c) 2013 Wesam Almobaideen et al. This is an open access article distributed under the Creative Commons Attribution License, which permits unrestricted use, distribution, and reproduction in any medium, provided the original work is properly cited.

\begin{abstract}
Mobile Ad-hoc Network (MANET) consists of mobile nodes that are connected via very dynamic multi-hop channels. Routing in MANET is a challenging task that has received great attention from researchers. In this paper we present Maximally Spatial Disjoint Multipath routing protocol (MSDM) which is a modification of AOMDV protocol. MSDM finds paths which are spatially separated and maximally disjointed. We think that sending various packets over spatially disjointed paths reduces the probability of collision occurrence and allows concurrent transmission over the set of different selected paths. Performance comparison of MSDM and AOMDV using GloMoSim simulator shows that MSDM is able to achieve a considerable improvement regarding some performance metrics such as delay, routing packets overhead, and network throughput.
\end{abstract}

Keywords: MANET; Routing Protocols; Multipath Routing; Spatial Disjoint Paths

\section{Introduction}

MANET is a collection of dynamic mobile nodes which are self-organized and able to communicate without using a preexisting network infrastructure. Each node acts as personal device and as a router and so it is able to forward data packet to other nodes. In mobile ad hoc networks the topology is very dynamic and changes frequently due to the nodes mobility. MANET's scarce resources such as battery based power and limited communications bandwidth make finding and maintaining a required route a key challenge. Proper design and selection of ad hoc routing protocols are needed to overcome these problems [1-3].

Many routing protocols have been proposed and evaluated to address challenges in MANET. These protocols can be generally classified as proactive (table-driven) routing protocols, reactive (on-demand), or hybrid of the world. In proactive routing protocols each node continuously maintains routing information about all other nodes in the network. These protocols incur considerable route maintenance overhead when compared with reactive protocols [1-3].

On demand (reactive) routing protocols are characterized with high route discovery latency. This is due to the fact that each node maintains routing information only when it needs to send data to a particular destination. The examples of a protocol that are based on reactive routing are the Dynamic Source Routing (DSR) [4], and Ad hoc On-demand Distance Vector Routing (AODV) [5]. These types of protocols may incur frequent route discovery requests if the topology is very dynamic [3].

Ad hoc routing protocols can be divided into Single path routing and multipath routing based on the number of discoverable paths between source and destination pairs of nodes. Multipath routing is preferred to reduce both the latency of discovering a new route after a link breakage and the control overhead. This is because route discovery procedures are only needed when all the discovered paths failed. Depending on the participating nodes (or links) in the path between two end nodes, multipath routing protocols can be node-disjoint or link-disjoint. For a particular source $S$, and destination $\mathrm{D}$, the set of node-disjoint routes consist of paths that do not have nodes present in more than one of S-D paths (except the source and destination). Similarly, the set of link-disjoint paths consist of paths that do not have certain link present in more than one of S-D paths [6].

Most of the existing multipath routing protocols are extensions of either AODV [5], or Dynamic Source 
Routing (DSR) [4]. Ad hoc On-demand Multipath Distance Vector (AOMDV) is a multipath extension of AODV that computes multiple loop-free link-disjoint routes [6]. Spilt Multi-path Routing (SMR) is a multipath routing protocol that modifies DSR by finding the set of maximally node-disjoint paths between a source and destination [7].

In this paper we are interested in multipath routing protocol, such as AOMDV protocol [6] and the potential improvement that could be achieved by choosing the most spatially separated disjoint paths, instead of choosing only disjointed ones. Sending various packets over spatially disjointed paths reduces the probability of collision occurrence that could otherwise happen when two nodes which belong to different non-spatially disjointed paths try to forward their packets simultaneously. This in turn, allows faster concurrent transmission over the set of different spatially disjointed paths.

The rest of this paper is organized as follows. The related work is presented in Section 2. Section 3 explains the proposed idea in more details. In Section 4 we show the results along with their evaluation and discussion. We conclude the paper in Section 5.

\section{Related Work}

In this section we present some related research to multipath routing in ad hoc networks. Lee and Gerla proposed an On-demand multipath routing protocol called Split Multipath Routing (SMR) in [7]. SMR is similar to DSR with a modified route request message that is used to find a number of maximally disjoint paths. An intermediate node forwards the received RREQs that have get through a different links. This RREQ should have a hop count that is not larger than the first received RREQ. The destination selects the route on which it has received the first RREQ packet as the shortest delay primary path. The destination node waits to receive multiple RREQs before selecting the path which is maximally disjoint compared with the primary path. Should the destination node discover more than one maximally disjoint path, it chooses those paths with the shortest hop count. Simulated evaluation results show that SMR outperforms DSR with fewer dropped packets and shorter end-to-end delay.

In [8], Meghanathan has presented a simulation-based analysis of the stability and hop count of node-disjoint and link-disjoint multi-path routes in mobile ad hoc networks. The results of the analysis have shown that nodedisjoint paths were as stable as link-disjoint paths and also there was not much difference in the hop count of these paths.

$\mathrm{Li}$ and Cuthbert proposed an extension of AODV called Node-Disjoint Multipath Routing protocol (NDMR) in [9]. NDMR modifies AODV to allow path accumulation feature existed in DSR during route request packet transmission in addition to discovering multiple node disjoint paths. Simulation results showed that NDMR has reduced routing overhead while increasing the packet delivery ratio.

A multipath extension to DSR is proposed in [10] to support multimedia applications. The proposed protocol called Shortest Multipath Source Routing (SMS). SMS builds multiple partial-disjoint paths from source to destination to reduce route discovery and to expedite recovery when a route is broken.

In [11] a new on-demand routing protocol called Spatially Disjoint Multipath Routing (SDMR) has been proposed. SDMR is capable of finding multiple paths in one route discovery. It then uses a developed metric to measure the distance between them. Finally, it chooses the most two separated paths. The new distance metric used to measure path separation proves to be congruent with Euclidean distance across nodes in the paths. A heuristic algorithm which, given a topology graph and utilizes the new distance metric, has been developed. This heuristic algorithm can discover spatially disjoint paths between source and destination nodes. The overhead of SMDR and OLSR has been evaluated both analytically, and by simulation. Results demonstrate the effectiveness of the protocol in finding spatially separated routes.

In [12] An Energy Aware Clustered-Based Multipath Routing (EACMR) has been proposed. EACMR forms several clusters and finds energy aware node-disjoint multiple routes. These routes are used to transmit data from a source to destination in order to increase the network life time.

Wesam AlMobaideen in [13] has presented a Stabilitybased Partially Disjoint AOMDV (SPDA) protocol which is a modification of the AOMDV protocol. SPDA finds partially disjoint paths based on links stability. The idea is that accepting partially disjointed paths that are more stable than other maximally disjoint ones could increase paths lifetime. This in turn improves MANET performance in terms of delay, routing packets overhead, and the network throughput.

In [14] an improved version of SPDA was proposed by adding the number of nodes each path passes through to the path selection criteria. In the Improved SPDA (ISPDA), the available alternative paths have been utilized to transmit packets in parallel. The authors have conducted several experiments to compare SPDA and ISPDA regarding the throughput and delay performance metrics. Results show that ISPDA outperforms SPDA in most of the adopted scenarios.

Grid-based Energy Aware Node-Disjoint Multipath Routing Algorithm has been proposed in [15]. This algorithm uses the same grid partitioning in the GRID routing 
protocol. The main difference between this algorithm and GRID routing protocols is that it considers energy-aware and node-disjoint multipath while GRID does not. Simulation results indicate that the proposed algorithm outperforms single path on-demand routing protocol such as AODV and DSR.

In [16], a new routing algorithm called Ant-based Energy Aware Disjoint Multipath Routing Algorithm (AEADMRA) has been developed. It is based on Ant colony algorithms which are subset of swarm intelligence. Ant colony algorithms are concerned with the ability of simple ants (software) to solve complex problems by cooperation. AEADMRA extends GRID in a way to enable path accumulation in route request/reply packets. This allows the discovery of multiple energy aware routing paths which are characterized with a low routing overhead. Simulation results show that AEADMRA performs much better than GRID.

In [17] a complete secure Multipath routing protocol (SecMR) was developed. SecMR works in two phases. The first phase is the neighboring authentication phase which is repeated in periodic time intervals so that to ensure the link-to-link authentication. In the second phase a signed request is generated by the source which gives the system an end-to-end authentication. Each intermediate node handles all the receiving requests in order to ensure that all possible node-disjoint paths can be finally found by the destination. SecMR is a multipath protocol in that it discovers all existing node-disjoint paths up to a given maximum number of hops.

\section{Description of MSDM Protocol}

AOMDV with link disjoint paths sends data packets through multiple paths which have no shared links. This speeds up data transmission by creating back up routes so that a break in one link affects only the path which contains that broken link [6]. In this paper, MSDM is presented as a modification to the AOMDV protocol. MSDM sends data packets through multiple paths which are the most spatially separated and node-disjoint paths.

In AOMDV, when a source needs a route to a destination, it starts route discovery process. Route discovery process is initiated by flooding Route Request (RREQ) packet across the network and waiting for Route Reply (RREP). Any intermediate node receiving a RREQ sets up a reverse path to the source and, if it has a valid route to the destination, it will generate a RREP to send it back to the requesting node. Otherwise, it will rebroadcast the RREQ packet. When the destination node receives a RREQ it generates a RREP and sends it back to the source node.

MSDM modifies AOMDV RREQ message to include the list of nodes participating in the path between a spe- cific source and destination. The inclusion of this list in the RREQ message helps in deciding whether a specific route satisfies the disjointness property or not. Figure 1 shows the RREQ packet used in MSDM.

MSDM utilizes additional two tables for proper operation. The first table is the Seen RREQ Table in which a node inserts information about any RREQ it overhears from its neighbors. This can be carried out since any node can put itself in the promiscuous mode to overhear messages sent by its neighbors. The second table is the Replied RREQ Table. This table is used by the node that generates a Route Reply (RREP) message to record the information about the route used in this reply.

While AOMDV discovers link-disjoint paths, MSDM on the other hand discovers a set of node-disjoint paths which are spatially separated. In MSDM, node disjointness of paths is achieved through modifying the RREQ packet to include the list of nodes participating in each path. Any intermediate node which decides to rebroadcast the RREQ must add its address to the Route List of the RREQ. Before generating a RREP a node must check the received Route-List in the RREQ to ensure that there are no common nodes between this path and the previously received paths which are stored in the Replied RREQ Table. If there is any common node between this path and other paths that have been accepted beforehand, the new path must be discarded.

Spatiality is achieved by filtering the RREQ packets received at intermediate nodes. Figure 2 presents the algorithm that does the filtering process. Any intermediate node receives a RREQ packet, checks the Seen RREQ Table to see if this RREQ was processed and rebroadcasted by any of its neighbors. If this is the case, it will not process and rebroadcast this route request unless it is the intermediate node which is the first node after the source or the last node before the destination.

This filtering technique adopted in MSDM reduces the overhead of processing and rebroadcasting RREQ packets

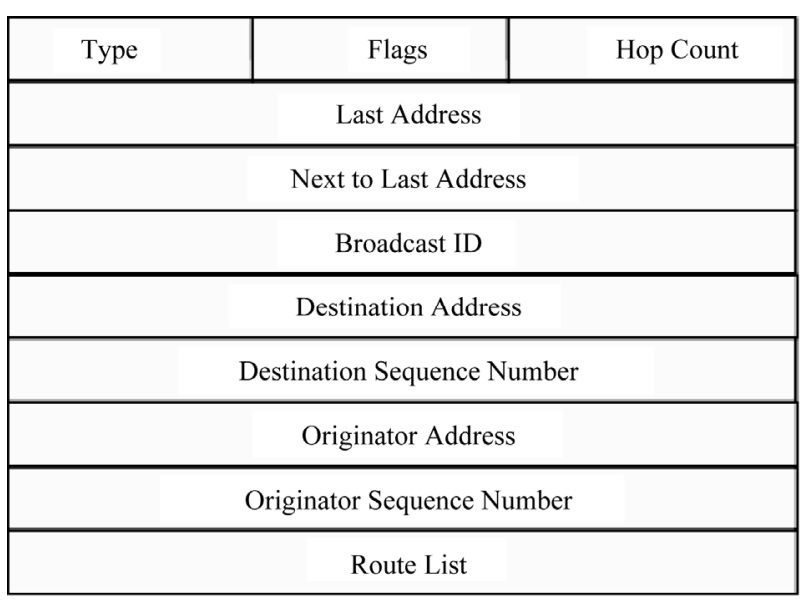

Figure 1. MSDM RREQ message format. 


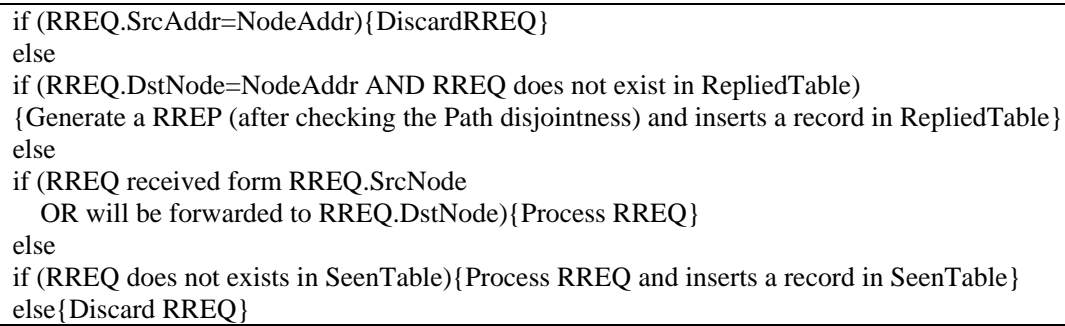

Figure 2. Node spatiality check algorithm.

which were processed by neighboring nodes. More importantly, this guarantees that RREQ packets reach the destination carrying spatially separated paths. Afterward, the sender can start to transmit its data concurrently over the set of spatially discovered paths. Transmitting data over such paths should reduce the collision overhead since the intermediate nodes are far away from each other, spatially disjoint, and so their transmission will not interfere.

In other words, spatially separated paths are discovered to ensure that data transmitted on one path will not interfere with transmissions on the other paths. This should reduce the probability of collisions that could occur between nodes trying to forward packets over different paths. This, consequently, increases the network throughput and reduces the end-to-end delay.

The reason of excluding nodes after the source and before the destination from processing more than one RREQ is to allow the protocol to discover as many as existed spatially separated paths. Otherwise, the number of discovered routes could be very limited. After receiving RREQ from these paths the destination chooses those paths that specify the node-disjoint criteria before sending a RREP over the reverse path associated with this RREQ.

MSDM is different from other protocols, which are also concerned with finding spatially disjoint paths and specifically, SDMR protocol proposed in [11] in various ways. Firstly, SDMR relies on the source to compute the set of spatially disjoint paths after it construct a graph that represents the network between the source and destination. MSDM, in contrast, uses a simpler filtering mechanism which enables the intermediate nodes between the source and destination to forward RREQ only through spatially disjoint paths. This means that the selection of spatially separated paths is done on the fly while the RREQ is being forwarded between the source and destination. Secondly, MSDM uses less complicated protocol which forwards RREQ that carries the ID of the set of nodes that constitute the path between source and the destination. The destination then computes the set of disjoint paths out of the received RREQs. SMDR, on the other hand, uses a more complicated algorithm that can be summarized in the following steps:

1) The source node floods RREQs which carry the ID of the set of intermediate nodes they will pass through in their way towards the destination node.

2) The destination has to find a subset of these paths that will be used to send RREPs back to the source.

3) The RREP is allowed to carry the 1-hop route information of each node in the revers path to the destination.

4) The source node is required to build a partial graph of the network by using the received neighborhood information.

5) Finally, the source node is asked to compute a set of candidate paths between source and destination before it chooses the pair of most disjoint paths.

Thirdly, SMDR forwards packets using source routing, while MSDM forwards packets using the usual way used in AODV and AOMDV.

\section{Performance Evaluation and Results Discussion}

We have evaluated the performance of MSDM and compared it with that of AOMDV using Global Mobile Simulation (GloMoSim) library [18]. Our simulation experiments modeled a network of 100 mobile hosts placed randomly within a $2000 \times 2000$ meters area. We have used the Distributed Coordination Function (DCF) of IEEE 802.11 for wireless LANs as the MAC layer protocol. In the experimental scenarios, the mobile nodes have been moving randomly for 400 seconds simulation time. Each node moved independently according to the random waypoint mobility model with $25(\mathrm{~m} / \mathrm{s})$ maximum mobility speed and 15 seconds as pause time. The simulated traffic was a Constant Bit Rate (CBR) with 10 connections. The size of application data packets was 512 bytes.

Each experiment has been repeated at least 25 times using different seeds and an average value of these runs has been computed to represent the final result of the measured performance metric. The average end-to-end delay, throughput, and the discovery overhead are the evaluated performance metrics. Traffic load, specified by the number of packets each node has to send, and the nodes density, specified by varying the simulated terrain area side length, have been chosen as the performance parameters. 
Next we present results and the discussion of the comparison between the proposed MSDM protocol and AOMDV regarding the above mentioned performance metrics and parameters. The next three experiments are concerned with measuring the performance of the two compared protocols while varying the traffic load. Afterwards, in the last three experiments, nodes density is considered as the performance parameter in this performance evaluation.

In order to change the traffic load of the network we increased the number of packets a traffic source node had to send varied from 20, 40, 60, 80 and up to 100 packets. Figure 3 shows the average end-to-end delay while changing the traffic load. One can notice that MSDM outperforms AOMDV almost for all the values of transmitted packet size. Moreover, the difference between the two protocols increases as the traffic load increases. This is due to the fact that MSDM uses spatially disjoint paths by which it avoids collisions between intermediate nodes trying to forward their packets.

The reduction of collisions in turn, reduces retransmissions of collide packets and as a result reduces the average end-to-end delay. This is in contrast with AOMDV which tries to choose disjoint paths and so concerned only with reducing the effect of link breakage by limiting its effects on only one path.

The second experiment presented in Figure $\mathbf{4}$ shows a comparison of the discovery overhead metric as the traffic load increases. The figure shows that as the traffic

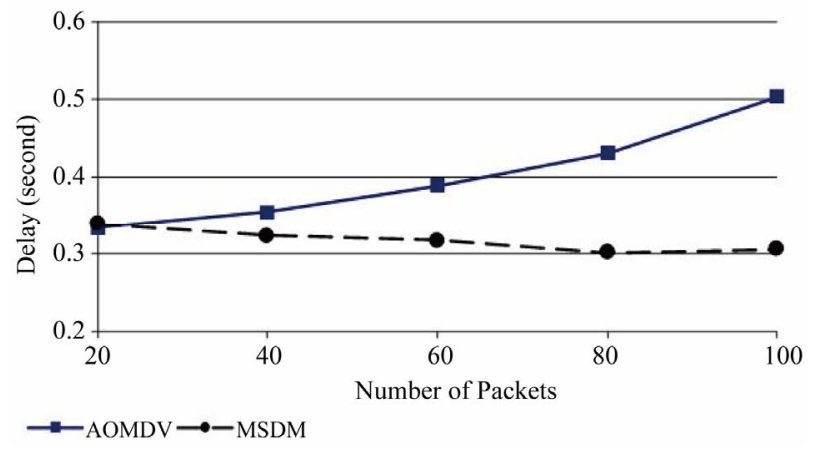

Figure 3. Average end-to-end delay vs number of packets.

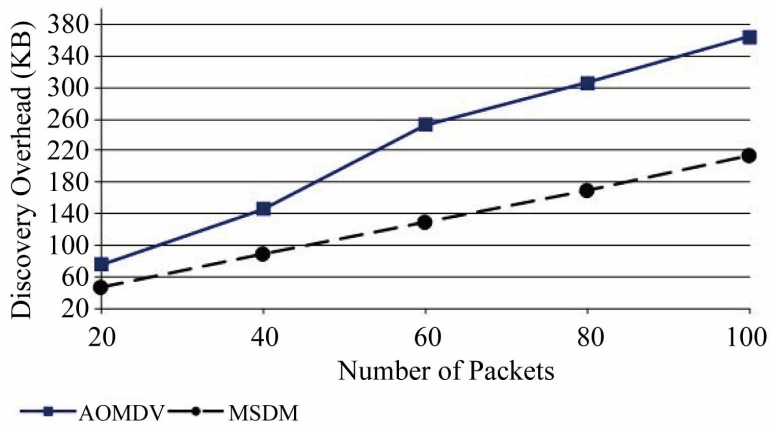

Figure 4. Discovery overhead vs number of packets. load increases, MSDM causes less discovery overhead than AOMDV. The gain in discovery overhead reduction is due to the filtering technique of RREQ packets. In MSDM, a node reduces the overhead of processing RREQ packets by discarding RREQ packets which were processed by that node's neighbors. AOMDV on the other hand, relies on the destination node in filtering the received RREQ by choosing the maximally disjoint ones. Filtering the RREQ on the destination node causes considerable overhead as the RREQ packet needs to go through the whole path towards the destination before being filtered.

Figure 5 presents results that are concerned with the network throughput performance metric. It is clear from the figure that MSDM outperforms AOMDV especially as the traffic load increases. The throughput of both MSDM and AOMDV decreases as the traffic load increases. The reduction in throughput as the traffic load gets higher happens due to the effect of more congestion level which reduced the number of delivered packets. The superiority of MSDM in throughput is due to the fact that it uses spatially node disjoint paths to send data packet.

Sending packets over spatially disjoint paths allows concurrent transmission over the set of different paths which in turn increases the network throughput. Forwarding packets over spatially separated paths reduces collisions between nodes that are member of the different paths which try to transmit over the shared medium. Maximally disjoint paths, adopted in AOMDV, do not allow that much of concurrency and reduction in collisions as spatially disjoint ones do.

In the next three experiments, nodes density is considered as the performance parameter in the evaluation of the two protocols regarding same performance metrics mentioned above. In order to change the density of nodes in the simulated terrain a gradual increment of the terrain area was done in order to move to a sparser node density mode. The successive experimental scenarios assume a square terrain with a side length ranging from 500, 1000, 1500, 2000, and up to 2500 meters.

The fifth experiment was conducted to compare the

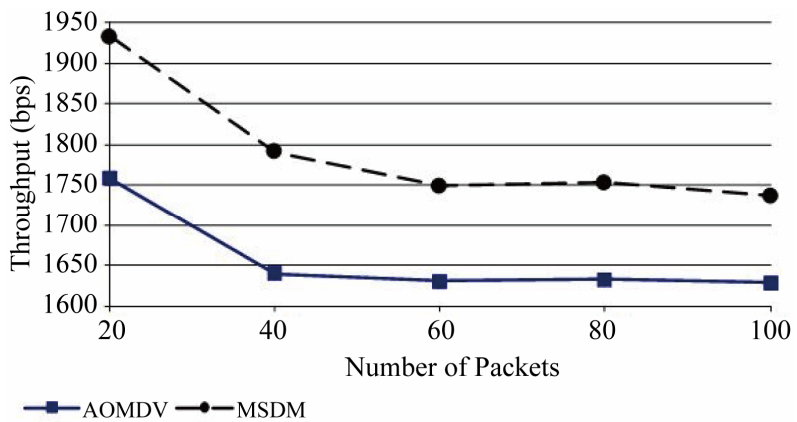

Figure 5. Throughput vs number of packets. 
end-to-end delay of MSDM and AOMDV while changing the nodes density based on the length of the square terrain side. The result of this experiment is shown in Figure 6. The figure illustrates that as the density of nodes decreases, MSDM incurs less delay compared with AOMDV. This is because, in contrast with AOMDV, MSDM chooses spatially node disjoint paths that allows intermediate nodes to send data without collisions. This is true for terrain side length above 1000 meters after which spatially disjoint paths can be easily found.

When the terrain side length becomes too much high, such as the value of 2500 and higher, we notice that the performance of the two protocols becomes comparable. This is because, given that the number of nodes remains fixed, it becomes harder for the two protocols to find multipaths when the nodes are highly scattered as it is the case in such a wide terrain areas.

Figure 7 shows a comparison of discovery overhead between MSDM and AOMDV. The discovery overhead of both MSDM and AOMDV increases as the density of nodes decreases. This can be explained by recalling that when nodes density becomes sparser the probability of path breakage becomes higher due to nodes mobility. Frequent path breakage will invoke the route discovery mechanism, which introduces overhead on network performance. We can notice from the Figure 7 that MSDM

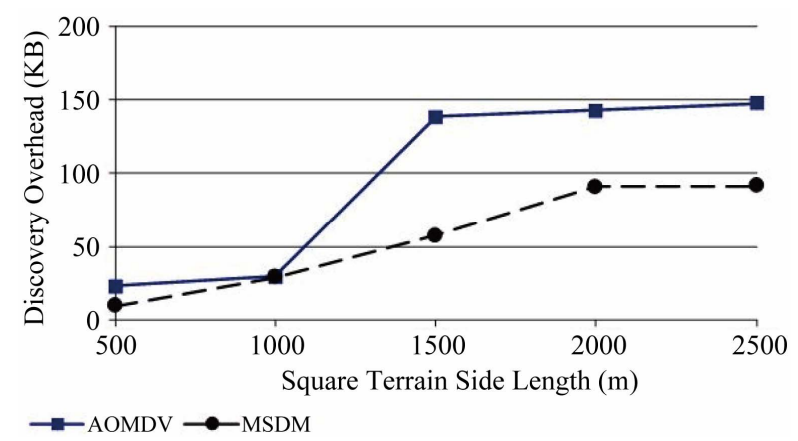

Figure 6. Average end-to-end delay vs terrain dimension (nodes density).

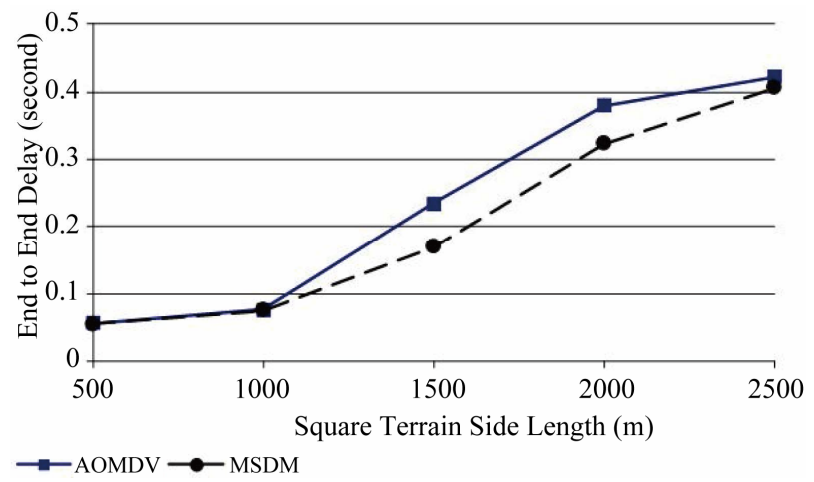

Figure 7. Discovery overhead vs terrain dimension (nodes density).

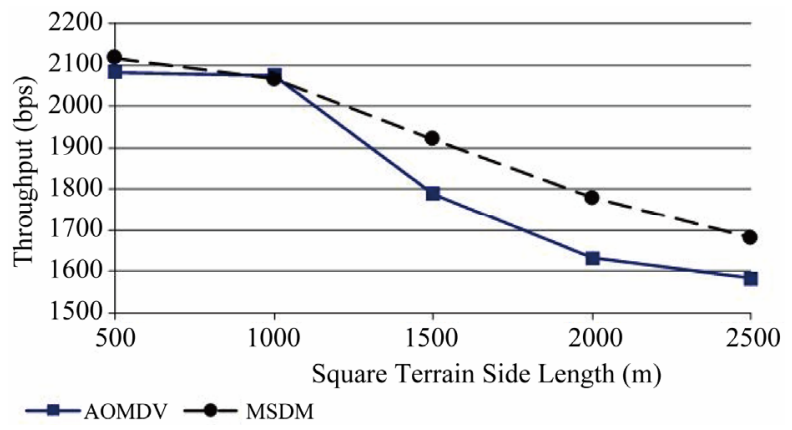

Figure 8. Throughput vs terrain dimension (nodes density).

has smaller discovery overhead than that of AOMDV since MSDM uses a filtering technique to prevent neighbors from processing the same RREQ. The effect of filtering RREQ while they are being forwarded towards the destination and even before being received by the destination becomes clearer as the nodes becomes sparser.

In Figure 8 we present a comparison of throughput between MSDM and AOMDV as the density of nodes decreases. We can notice from the figure that MSDM achieves higher throughput than AOMDV as the nodes became sparser. Figure 8 also shows that the throughput of both protocols decreases as the nodes became sparser. This is because when the nodes became sparser, path breakage probability becomes greater which reduces the number of concurrent paths used. MSDM still perform better than AOMDV since it tries to avoid spatially close paths which have higher probability to be affected by nodes mobility.

\section{Conclusions}

In this paper we have presented MSDM protocol as a modification to the AOMDV. MSDM chooses paths which are spatially separated and node-disjoint. Selecting spatially node-disjoint routes improves the network performance when compared with AOMDV which chooses link-disjoint paths only.

The throughput, average end to end delay, and route discovery overhead performance metrics have been measured for MSDM and AOMDV. Results have shown that MSDM out-performs AOMDV with regard to those performance metrics while varying traffic load and nodes density. As the traffic load of the network increases, the superiority of MSDM becomes clearer.

\section{REFERENCES}

[1] M. Abolhasan, T. Wysocki and E. Dutikiewwicz, "A Review of Routing Protocols for Mobile Ad Hoc Networks,” Journal of Ad Hoc Networks, Vol. 2, No. 1, 2004, pp. 1-22.

http://dx.doi.org/10.1016/S1570-8705(03)00043-X

[2] K. Sahadevaiah and O. B. Ramanaiah, “An Empirical 
Examination of Routing Protocols in Mobile Ad Hoc Networks," International Journal of Communications, Network and System Sciences, 2010, Vol. 3, No. 6, pp. 511-522. http://dx.doi.org/10.4236/ijcns.2010.36069

[3] W. A. AlMobaideen, H. M. Mimi, F. A. Masoud and E. Qaddoura, "Performance Evaluation of Multicast Ad Hoc On-Demand Distance Vector Protocol,” Journal of Computer Communications, Vol. 30, No. 9, 2007, pp. 19311941.

[4] D. B. Johnson, D. A. Maltz and J. Broch, "Dynamic Source Routing in Ad Hoc Wireless Networks," Mobile Computing, The Kluwer International Series in Engineering and Computer Science, Vol. 353, 1996, pp. 153181.

[5] C. E. Perkins and E. M. Royer, "Ad Hoc On-Demand Distance Vector Routing,” Proceedings of the 2nd Annual IEEE International Workshop on Mobile Computing Systems and Applications, New Orleans, 25-26 February 1999, pp. 90-100.

[6] M. K. Marina and S. R. Das, "On-Demand Multipath Distance Vector Routing in Ad Hoc Networks," Proceedings of IEEE International Conference on Network Protocols (ICNP), Riverside, 11-14 November 2001, pp. 14-23.

[7] S.J. Lee and M. Gerla, "Split Multipath Routing with Maximally Disjoint Paths in Ad Hoc Networks," IEEE International Conference On Communications, (ICC 2001), Helsinki, 11-14 June 2001, pp. 3201-3205.

[8] N. Meghanathan, "Stability and Hop Count of NodeDisjoint and Link-Disjoint Multi-path Routes in Ad Hoc Networks," Proceedings of the Third IEEE international Conference on Wireless and Mobile Computing, Networking and Communications, White Plains, 8-10 October 2007, p. 42.

[9] X. Li and L. Cuthbert, "On-Demand Node-Disjoint Multipath Routing in Wireless Ad hoc Networks,” Proceedings of the 29th Annual IEEE International Conference on Local Computer Networks (LCN'04), Tampa, 16-18 November 2004, pp. 419-420.

[10] H. Zafar, D. Harle, I. Andonovic and M. Ashraf, "PartialDisjoint Multipath Routing for Wireless Ad-Hoc Net- works," Proceedings of the 32nd IEEE Conference on Local Computer Networks, Dublin, 15-18 October 2007, pp. 258-259.

[11] J. J. Gálvez, P. M. Ruiz and A. F. G. Skarmeta, "Multipath Routing with Spatial Separation in Wireless MultiHop Networks without Location Information,” The International Journal of Computer and Telecommunications Networking, Vol. 55, No. 3, 2011, pp. 583-599.

[12] M. Bheemalingaiah, M. M. Naidu and D. Sreenivasa Rao, "Energy Aware Clustered Based Multipath Routing in Mobile Ad Hoc Networks," International Journal of Communications, Network and System Sciences, Vol. 2, No. 2, 2009, pp. 123-130.

[13] W. AlMobaideen, "SPDA: Stability Based Partially Disjoint AOMDV," European Journal of Scientific Research, Vol. 27, No. 3, 2009, pp.342-348.

[14] W. Almobaideen, D. Al-Khateeb, A. Sleit, M. Qatawneh, R. Al-Khdour and H. Abu Hafeeza, "Improved Stability Based Partially Disjoint AOMDV,” International Journal of Communications, Network and System Sciences, Vol. 6, No. 5, 2013, pp. 244-250. http://dx.doi.org/10.4236/ijcns.2013.65027

[15] Z. Wu, X. Dong and L. Cui, “A Grid-Based Energy Aware Node-Disjoint Multipath Routing Algorithm for MANETs," Proceedings of the Third international Conference on Natural Computation (ICNC 2007), Haikou, 24-27 August 2007, pp. 244-248.

[16] Z. Y. Wu, S. F. Jiang and X. M. Xu, "Ant-Based Energy Aware Disjoint Multipath Routing Algorithm in MANETs," Computer Journal, Vol. 53, No. 2, 2008, pp. 166-176. http://dx.doi.org/10.1093/comjnl/bxn007

[17] R. Mavropodi, P. Kotzanikolaou and C. Douligeris, "SecMR-A Secure Multipath Routing Protocol for Ad Hoc Networks," Ad Hoc Networks, Vol. 5, No. 1, 2007, pp. 87-99. http://dx.doi.org/10.1016/j.adhoc.2006.05.020

[18] X. Zeng, R. Bagrodia and M. Gerla, "GloMoSim: A Library for Parallel Simulation of Large-Scale Wireless Networks, Pads," Proceedings of 12th Workshop on Parallel and Distributed Simulation (PADS’98), Banff, 26-29 May 1998, p. 154. 